Journal of Chromatography, 89 (1974) 87-91

(C) Elsevier Scientific Publishing Company, Amsterdam - Printed in The Netherlands

CHROM. 7114

Note

\title{
A novel method for the separation and identification of bile acids and phos- pholipids of bile on thin-layer chromatograms
}

\section{S. K. GOSWAMI and C. F. FREY}

Department of Surgery, Wayne Coumty General Hospital, Eloise, Mich. 48132 (U.S.A.) and University of Michigan Medical Center, Ann Arbor, Mich. 48104 (U.S.A.)

(Received August 27th, 1973)

Cholesterol gallstone disease, so common in the western world, has consideraile medical and economic importance. Under normal physiological conditions, cholesterol is held in solution in the bile. If the solubility of cholesterol in bile is changed, cholesterol crystals will precipitate and lead to the formation of gallstones. The solubility of cholesterol in bile depends on three major lipid components of bile: conjugated bile salts, phospholipids and cholesterol ${ }^{1}$. Knowledge of bile lipid composition is important in our understanding of the mechanism of gallstone formation.

Paper chromatography ${ }^{2}$ and silicic acid column chromatography ${ }^{3}$ have been used for the analysis of the composition of bile lipids. However, the lat ter technique has been found less satisfactory for routine bile analysis or to experiment on small animals because of the limited quantity of the bile samples. Although thin-layer chromatographic separation of bile lipids ${ }^{4}$ can be performed using three successive solvent systems, it takes as long as $8 \mathrm{~h}$ and does not delineate the free bile acids one from the other. Since bile contains a variety of compounds, different plates and different solvent systems must be used for the separation of each class of compounds. Various solvent systems have been proposed to achieve the separation of bile acids and their conjugates ${ }^{5,6}$ on thin-layer chromatograms. Our method successfully accomplishes the separation of several classes of bile lipids on a single plate which makes a direct comparison possible between different bile lipid components such as cholesterol, free and conjugated bile acids, lecithin and lysolecithin. The solvent system and the spray reagent used in our technique, not previously described, are satisfactory for a routine check of bile lipid composition and may be used for the separation of individual bile acids, both free and conjugated, in biological fluids on the same plate.

\section{MATERIALS AND METHODS}

\section{Chemicals}

Cholesterol, deoxycholic acid, chenodeoxycholic acid, taurodeoxycholic acid, glyco- and taurochenodeoxycholic acid, glycocholic acid, lysolecithin (all from Sigma, St. Louis, Mo., U.S.A.) cholic acid and lithocholic acid (Applied Science Labs., State College, Pa., U.S.A.), lecithin and taurocholic acid (Nutritional Bio- 
chemicals, Cleveland, Ohio, U.S.A.), glycodeoxycholic acid (Calbiochem., San Diego, Calif., U.S.A.), isopropyl alcohol, acetic acid, sulphuric acid (Baker Analyzed Reagent, J. T. Baker, Phillipsburg, N.J., U.S.A.), ammonium molybdate, ethyl acetate, isooctane (Mallinckrodt, St. Louis, Mo., U.S.A.) chloroform, methanol (Matheson, Coleman and Bell, Norwood, Ohio, U.S.A.) were used.

Bile samples were collected by puncture of the gallbladder during operation and when the gallbladder was removed, the total amount of bile was taken, frozen and stored at $-16^{\circ}$.

\section{Preparation of spray reagent}

The spray reagent was the same as used for phospholipid detection? ${ }^{7}$. A piece of copper wire is placed in a solution of $0.5 \mathrm{~g}$ of ammonium molybdate in $2 \mathrm{ml}$ of distilled water. The mixture is chilled and $2 \mathrm{ml}$ of concentrated sulphuric acid are added; the deep blue solution is then shaken. This reaction mixture is kept for $2 \mathrm{~h}$ at room temperature with occasional shaking. A $80-\mathrm{ml}$ volume of distilled water is then added and shaken; the color changes from deep blue to light brown, the copper wire is then removed and $6.4 \mathrm{ml}$ of concentrated sulphuric acid are added and mixed; the resulting solution remains light brown. This reagent can be stored for a week or more in the refrigerator.

\section{Experimental}

The solutions to be tested were applied on $20 \times 20 \mathrm{~cm}$ pre-coated thin-layer plate silica gel $\mathrm{F}_{254}$ of $0.25 \mathrm{~mm}$ thickness (E. Merck, Darmstadt, G.F.R. distributed by Brinkmann, Westbury, N.Y., U.S.A.). The plate was marked at $6 \mathrm{~cm}$ and $13 \mathrm{~cm}$ from the starting line. About 10 $\mu$ g samples of each of free and conjugated bile acids and phospholipids were applied as spots of $4 \mathrm{~mm}$ diameter at $1.5 \mathrm{~cm}$ above the bottom edge of the plate. Volumes of 0.5-1.0 $\mu$ l of the bile samples were applied directly to the plate using a Hamilton microliter syringe. When samples are applied, the plate must not be dried with hot air as some of the bile acids might be adsorbed to the silica gel and might not move in the chromatographic system. The plate was then placed in a commercial chromatographic chamber (Gelman. Ann Arbor, Mich., U.S.A.) which was previously saturated with the solvent system isooctane-isopropyl alcohol-ethyl acetate-acetic acid $(40: 20: 10: 10, \mathrm{v} / \mathrm{v})$ at room temperature. After the center of the solvent front reached the $13-\mathrm{cm}$ mark, the plate was taken out of the chamber and dried with cold air for 2-3 min by means of a hair-drier. The plate is next placed in the second chamber and developed with the solvent system chloroform-methanol-water $(65: 35: 4, v / v)$ up to the $6-\mathrm{cm}$ mark and taken out of the chamber.

The plate was then kept at $70-80^{\circ}$ in an oven for $1-2 \mathrm{~min}$, taken out of the oven and sprayed with the spraying reagent. The plate was then heated in the oven at $70-80^{\circ}$ for $5-7 \mathrm{~min}$ and the color of cholesterol and phospholipids noticed and the area marked. The plate is allowed to heat again for another 5-7 min and the color of bile acids noted.

\section{RESULTS AND DISCUSSION}

Bile samples from gallstone patients and pure bile acids, conjugated and 
TABLE I

THE $R_{p}$ VALUES OF BILE ACIDS, PHOSPHOLIPIDS AND CHOLESTEROL AND THE COLOR PRODUCED BY THE NEW SPRAY REAGENT

The $R_{r}$ values are represented as percent of the distance of solvent front from the origin and the $R_{a t}$ values as percent of the distance of cholesterol from the origin.

\begin{tabular}{lccl}
\hline Substance & $R_{r}$ & $R_{\boldsymbol{r}}$ & Color \\
\hline Lysolecithin & 2.6 & 3 & Blue \\
Lecithin & 7.2 & 8.2 & Blue \\
Taurocholic acid & 14 & 15.6 & Greyish green \\
Taurochenodeoxycholic acid & 20 & 23 & Purple \\
Taurodeoxycholic acid & 21 & 24 & Greyish green \\
Glycocholic acid & 34 & 36.6 & Greyish green \\
Glycochenodeoxycholic acid & 55 & 63 & Purple \\
Glycodeoxycholic acid & 56 & 64 & Greyish green \\
Cholic acid & 71 & 80 & Greenish yellow \\
Chenodeoxycholic acid & 78 & 89 & Purple \\
Deoxycholic acid & 81 & 92 & Yellow \\
Lithocholic acid & 85 & 96 & Light purple \\
Cholesterol & 88 & 100 & Pink, turns greenish grey \\
\hline
\end{tabular}

unconjugated, cholesterol, lysolecithin and lecithin were examined by this method. The $R_{F}$ values and the color produced by each component are shown in Table $I$. The $R_{F}$ values presented are means of at least five runs and they possibly apply to the particular batches of plates employed and hence are not an absolute value but would be expected to vary from one individual to another. It is suggested each investigator will want to prepare his own color standard and the $R_{F}$ values.

The solvent front forms a concave shape at the end of the run and a solvent demixing occurs at the center of the plate showing disproportionate $R_{F}$ values. Hence a mixture of known compounds was spotted between every fourth sample. The important feature of this new spray reagent is that, whatever might be the difference of $R_{k}$ values, the compounds tested can be identified by their characteristic colors which are very distinct amongst ench other. The blue phospholipid color and the pink cholesterol color appear within 5-7 min of heating in the oven, other bile acid colors appeared after that. All the colors stand out clearly in an almost white background. Various spray reagents have been $u \mathrm{us}^{8-11}$ in the past for the detection of bile acids on thin-layer chromatograms; the present reagent is a unique and useful addition.

The solvent systems previously ${ }^{12,13}$ used for the separation of bile acids, conjugated and unconjugated, separate either the free bile acids or the conjugated bile acids but not all on the same plate, nor do they separate chenodeoxycholic acid from deoxycholic acid. The solvent systems that we have developed completely separate the conjugated bile acids from free bile acids leaving each component distinct and separate in the same plate, e.g. chenodeoxycholic acid is separated from deoxycholic acid. The dihydroxybile acid-taurine conjugates, taurodeoxycholic and taurochenodeoxycholic acid, have the same mobility, as do the dihydroxybile acidglycine conjugates, glycodeoxycholic acid and glycochenodeoxycholic acid, but the 
presence of both chenodeoxy- and deoxycholic acid conjugates in the same sample can be recognized by a mixed color of the spot i.e. greenish purple.

The main advantage of this technique for the separation of bile is its accomplishment on a single plate using only a small amount of $0.5 \mu \mathrm{l}$ of bile, and the rapid completion of the operation within $4 \mathrm{~h}$. Part of the bile pigment migrated with the solvent, while the rest stayed on the starting point, and did not interfere with the color development and identification of the bile salts. Thus this micro-technique can be used for routine bile analysis and permits the direct study of different components of bile important in gallstone formation. Furthermore, this method does not involve preliminary extraction and separation of each class of bile lipids, which is a part of the usual bile lipids analysis.

Thirty samples of gallbladder bile from gallstone patients were examined by this technique. Glycine- and taurine-conjugated cholic and chenodeoxycholic acid, lecithin and cholesterol were present in all of the samples tested. Lysolecithin and glycodeoxycholic acid were found in ten samples. No taurine-conjugated deoxycholic acid was found on the chromatogram by this method. Free bile acids are also absent in the bile which supports previous observations ${ }^{14.15}$. The presence of lecithin and lysolecithin in bile has been reported earlier ${ }^{16}$. The absence or presence of a trace amount of glycodeoxycholic acid in the bile of patients with gallstones may be the result of interrupting the enterohepatic circulation, as denxycholic acid is formed by bacterial action on cholic acid during the enterohepatic circulation ${ }^{17-19}$. Deoxycholic acid was also found to be absent in patients with acute hepatitis, biliary dyskinesia and portal cirrhosis ${ }^{15}$. The explanation for the absence of taurine conjugation of deoxycholic acid in gallstone patients is obscure.

\section{ACKNOWLEDGEMENT}

The authors wish to acknowledge the assistance given by Mrs. S. Goswami during the earlier part of this experiment. This investigation was aided by a grant from the Medical Staff Research and Education Fund, Wayne County General Hospital.

\section{REFERENCES}

1 D. M. Small, Ade. Intern. Med., 16 (1970) 243.

2 J. Sjövall, Clin. Chim. Acta, 4 (1959) 652.

3 F. Nakayama and C. G. Johnston, J. Lab. Clin. Med., 59 (1962) 364.

4 F. Nakayama, M. Oishi, N. Sakaguchi and H. Miyake, Clin. Chim. Acta, 10 (1964) 544.

5 A. F. Hofmann, in A. T. James and L. J. Morris (Editors), New Biochemical Separations, Van Nostrand, London, 1964, p. 261.

6 P. Eneroth, J. Lipid Res., 4 (1963) 11.

7 S. K. Goswami and C. F. Frey, J. Lipid Res., 12 (1971) 509.

8 S. K. Goswami and C. F. Frey, J. Chromatogr., 47 (1970) 126.

9 S. K. Goswami and C. F. Frey, J. Chromatogr., 53 (1970) 389.

10 D. Kritchevsky, D. S. Martak and G. H. Rothblat, Anal. Biochem., 5 (1963) 388.

11 W. L. Anthony and W. T. Beher, J. Chromatogr., 13 (1964) 567.

12 A. F. Hofmann, J. Lipid Res., 3 (1962) 127.

13 M. A. Eastwood, K. Hamilton and L. Mowbray, J. Cliromatogr., 65 (1972) 407.

14 J. C. Erkanz and J. Sjövall, Acra Chem. Scand., 11 (1957) 1093.

15 J. Sjövall, Clin. Chim. Acta, 5 (1960) 33. 
16 A. Gottfries, S. Nilsson, B. Samuelsson and T. Schersten. Scand. J. Clin. Lab. Invest., 21 (1968) 168.

17 P. H. Ekdahl and J. Sjövall, Acra Chir. Scand., 114 (1957) 439.

18 S. Lindstedt, Ark. Keml, 11 (1957) 145.

19 A. Norman and J. Sjövall, J. Blol. Chem., 233 (1958) 872. 